\title{
INFINITESIMAL AND LOCAL RIGIDITY OF MAPPINGS OF CR MANIFOLDS
}

\author{
GIUSEPPE DELLA SALA, BERNHARD LAMEL, AND MICHAEL REITER
}

\begin{abstract}
A holomorphic mapping $H$ between two real-analytic CR manifolds $M$ and $M^{\prime}$ is said to be locally rigid if any other holomorphic map $F: M \rightarrow M^{\prime}$ which is close enough to $H$ is obtained by composing $H$ with suitable automorphisms of $M$ and $M^{\prime}$. With the aim of reducing the local rigidity problem to a linear one, we provide sufficient infinitesimal conditions. Furthermore we study some topological properties of the action of the automorphism group on the space of nondegenerate mappings from $M$ to $M^{\prime}$.
\end{abstract}

\section{INTRODUCTION}

Let $M \subset \mathbb{C}^{N}$ be a (real-analytic) generic submanifold. We define $\operatorname{Aut}_{0}(M)$ to be the group of the germs $\sigma$ of biholomorphic maps $\mathbb{C}^{N} \rightarrow \mathbb{C}^{N}$, defined around 0 , such that $\sigma(0)=0$ and $\sigma(M) \subset M$. We denote the Lie algebra of $\operatorname{Aut}_{0}(M)$ by $\mathfrak{h o l}_{0}(M)$.

Let now $M$ and $M^{\prime}$ be germs of generic real-analytic CR submanifolds in $\mathbb{C}^{N}$ and $\mathbb{C}^{N^{\prime}}$ respectively, and let $\mathcal{H}\left(M, M^{\prime}\right)$ denote the space of germs of holomorphic mappings which send $M$ into $M^{\prime}$. The group $G=\operatorname{Aut}_{0}(M) \times \operatorname{Aut}_{0}\left(M^{\prime}\right)$, which we call the isotropy group, acts on $\mathcal{H}\left(M, M^{\prime}\right)$ via $H \mapsto \sigma^{\prime} \circ H \circ \sigma^{-1}$, where $\left(\sigma, \sigma^{\prime}\right) \in G$ and $H \in \mathcal{H}\left(M, M^{\prime}\right)$. If we endow all of these sets with their natural (inductive limit) topologies, they become topological spaces and groups, respectively. We are interested in studying the topological properties of this (continuous) group action. More precisely we would like to continue our study of local rigidity of mappings, a notion we introduced in [7]: we say a map $H \in \mathcal{H}\left(M, M^{\prime}\right)$ is locally rigid if it projects to an isolated point in the quotient $\mathcal{H}\left(M, M^{\prime}\right) / G$ (for a formal definition, see Definition 44).

Our aim is to provide linear - and thus easier to compute - sufficient conditions for local rigidity. In order to state a criterion in this direction, we say a holomorphic section $V$ of $\left.T^{1,0}\left(\mathbb{C}^{N^{\prime}}\right)\right|_{H\left(\mathbb{C}^{N}\right)}$, which vanishes at 0 , is an infinitesimal deformation of $H \in \mathcal{H}\left(M, M^{\prime}\right)$ if $\operatorname{Re} V$ is tangent to $M^{\prime}$ along $H(M)$ (for the formal definition see Definition [11). We denote the set of infinitesimal deformations of $H$ by $\mathfrak{h o l}_{0}(H)$; it forms a real vector space.

2010 Mathematics Subject Classification. Primary 32H02; Secondary 32V40, 58E40.

The first author was supported by the FWF project P24878-N25, and would also like to thank the Center for Advanced Mathematical Sciences (CAMS) at AUB. The second author was supported by the FWFProject I382 and QNRF-Project NPRP 7- 511-1-098. The third author was supported by the FWF-Project P28873. 
We are particularly interested in sets of mappings satisfying certain generic nondegeneracy conditions introduced in [13]: in Definition 3 below we formally introduce the set of finitely nondegenerate mappings.

We are now going to state our main results, which are built on the approach and techniques of our recent work [7. In this paper we exploit our method of infinitesimal deformations to study more general situations. The first result is a generalization of Theorem 1 of [7].

Theorem 1. Let $M$ be a germ of a generic minimal real-analytic submanifold through 0 in $\mathbb{C}^{N}$, and $M^{\prime}$ be a germ of a generic real-analytic submanifold in $\mathbb{C}^{N^{\prime}}$. Let $H \in \mathcal{H}\left(M, M^{\prime}\right)$ be a germ of a finitely nondegenerate map satisfying $\operatorname{dim}_{\mathbb{R}} \mathfrak{h o l}_{0}(H)=0$. Then $H$ is an isolated point in $\mathcal{H}\left(M, M^{\prime}\right)$, and in particular, $H$ is locally rigid.

In the next result we are going to relax the assumption $\operatorname{dim}_{\mathbb{R}} \mathfrak{h o l} \mathfrak{l}_{0}(H)=0$. In order to do so we will restrict to the case when $M \subset \mathbb{C}^{N}$ and $M^{\prime} \subset \mathbb{C}^{N^{\prime}}$ are strictly pseudoconvex hypersurfaces, so that $M$ and $M^{\prime}$ have CR dimension $n=N-1$ and $n^{\prime}=N^{\prime}-1$ respectively. Moreover, since the embeddings of spheres have been studied extensively [6, 8, 11, 14, 17] (see [7] for a more thorough discussion and additional references), we assume that $M$ or $M^{\prime}$ is not biholomorphically equivalent to a sphere.

The following result is a generalization of Theorem 2 of [7] in the setting of strictly pseudoconvex hypersurfaces.

Theorem 2. Let $M \subset \mathbb{C}^{N}$ and $M^{\prime} \subset \mathbb{C}^{N^{\prime}}$ be germs of strictly pseudoconvex real-analytic hypersurfaces through 0 , where at least one of $M$ or $M^{\prime}$ is not spherical. If $H \in \mathcal{H}\left(M, M^{\prime}\right)$ is a germ of a 2-nondegenerate map that satisfies $\operatorname{dim}_{\mathbb{R}} \mathfrak{h o l}_{0}(H)=\operatorname{dim}_{\mathbb{R}} \mathfrak{h o l}_{0}\left(M^{\prime}\right)$, then $H$ is locally rigid.

We note that the assumption of 2-nondegeneracy implies that $N^{\prime} \leq \frac{N(N+1)}{2}$.

The outline of the paper is as follows: The proofs of our main results are provided in the very last section [6. Before, we fix notation in section 2 and give a jet parametrization result for finitely nondegenerate maps in section 3 . Then, we study infinitesimal deformations in section 4 and deduce some crucial properties of the action of isotropies on the space of maps in section 5 .

\section{Preliminaries}

This section is devoted to introduce some standard notation. For details and proofs, we refer the reader to e.g. [2].

For a generic real-analytic $\mathrm{CR}$ submanifold $M \subset \mathbb{C}^{N}$ we denote by $n$ its $\mathrm{CR}$ dimension and by $d$ its real codimension so that $N=n+d$. It is well-known (cf. [2]) that one can choose normal coordinates $(z, w) \in \mathbb{C}_{z}^{n} \times \mathbb{C}_{w}^{d}=\mathbb{C}^{N}$ such that the complexification $\mathcal{M} \subset \mathbb{C}_{z, \chi, w, \tau}^{2 N}$ of $M$ is given by

$$
w=Q(z, \chi, \tau), \quad(\text { equivalently }: \tau=\bar{Q}(\chi, z, w)),
$$

for a suitable germ of holomorphic map $Q: \mathbb{C}^{2 n+d} \rightarrow \mathbb{C}^{d}$ satisfying the following equations

$$
Q(z, 0, \tau) \equiv Q(0, \chi, \tau) \equiv \tau, \quad Q(z, \chi, \bar{Q}(\chi, z, w)) \equiv w .
$$


Given a defining equation $\rho^{\prime} \in\left(\mathbb{C}\left\{Z^{\prime}, \bar{Z}^{\prime}\right\}\right)^{d^{\prime}}$ for $M^{\prime}$, we recall that a germ of a mapping $H(z, w) \in(\mathbb{C}\{z, w\})^{N^{\prime}}$ with $H(0,0)=0$, belongs to $\mathcal{H}\left(M, M^{\prime}\right)$ if and only if it solves the mapping equation

$$
\rho^{\prime}(H(z, w), \bar{H}(\chi, \tau))=0 \quad \text { for } w=Q(z, \chi, \tau) .
$$

We endow $(\mathbb{C}\{Z\})^{N^{\prime}}$, which we consider as the space of germs at 0 of holomorphic maps from $\mathbb{C}^{N}$ to $\mathbb{C}^{N^{\prime}}$, with the natural direct limit topology and consider $\mathcal{H}\left(M, M^{\prime}\right) \subset(\mathbb{C}\{Z\})^{N^{\prime}}$ with the induced topology.

We denote by $L_{j}$ and $\bar{L}_{j}, j=1, \ldots, n$, a commuting basis of the germs of $\mathrm{CR}$ and anti-CR vector fields, respectively, tangent to $\mathcal{M}$. Furthermore it will be convenient to consider the following vector fields, which are also tangent to $\mathcal{M}$ :

$$
T_{\ell}=\frac{\partial}{\partial w_{\ell}}+\sum_{k=1}^{d} \bar{Q}_{w_{\ell}}^{k}(\chi, z, w) \frac{\partial}{\partial \tau_{k}}, \quad S_{j}=\frac{\partial}{\partial z_{j}}+\sum_{k=1}^{d} \bar{Q}_{z_{j}}^{k}(\chi, z, w) \frac{\partial}{\partial \tau_{k}}
$$

where $1 \leq \ell \leq d, 1 \leq j \leq n$.

In the sequel we denote by $\mathbb{N}=\{1,2,3, \ldots\}$ the set of natural numbers and write $\mathbb{N}_{0}=\mathbb{N} \cup\{0\}$. The notion of nondegeneracy we are interested in was introduced in [13]. For our purposes we will also need a slightly weaker one:

Definition 3. Let $M^{\prime}=\left\{\rho^{\prime}=0\right\}$, where $\rho^{\prime}=\left(\rho_{1}^{\prime}, \ldots, \rho_{d^{\prime}}^{\prime}\right) \in\left(\mathbb{C}\left\{Z^{\prime}, \zeta^{\prime}\right\}\right)^{d^{\prime}}$ is a local defining function for $M^{\prime}$. Given a holomorphic map $H=\left(H_{1}, \ldots, H_{N^{\prime}}\right) \in(\mathbb{C}\{z, w\})^{N^{\prime}}$, a fixed sequence $\left(\iota_{1}, \ldots, \iota_{N^{\prime}}\right)$ of multiindices $\iota_{m} \in \mathbb{N}_{0}^{n}$ and integers $\ell^{1}, \ldots, \ell^{N^{\prime}}$ with $1 \leq \ell^{j} \leq d^{\prime}$, we consider the determinant

$$
s=\operatorname{det}\left(\begin{array}{ccc}
L^{\iota_{1}} \rho_{\ell^{1}, Z_{1}^{\prime}}^{\prime}(H(z, w), \bar{H}(\chi, \tau)) & \cdots & L^{\iota_{1}} \rho_{\ell^{1}, Z_{N^{\prime}}^{\prime}}^{\prime}(H(z, w), \bar{H}(\chi, \tau)) \\
\vdots & \ddots & \vdots \\
L^{\iota}{ }_{N^{\prime}} \rho_{\ell^{N^{\prime}}, Z_{1}^{\prime}}^{\prime}(H(z, w), \bar{H}(\chi, \tau)) & \cdots & L^{\iota_{N^{\prime}}} \rho_{\ell^{N^{\prime}}, Z_{N^{\prime}}^{\prime}}^{\prime}(H(z, w), \bar{H}(\chi, \tau))
\end{array}\right) .
$$

We define the open set $\mathcal{F}_{k} \subset \mathcal{H}\left(M, M^{\prime}\right)$ as the set of maps $H$ for which there exists a sequence of multiindices $\left(\iota_{1}, \ldots, \iota_{N^{\prime}}\right)$ with $k=\max _{1 \leq m \leq N^{\prime}}\left|\iota_{m}\right|$ and integers $\ell^{1}, \ldots, \ell^{N^{\prime}}$ as above such that $s(0) \neq 0$. We will say that $H$ with $H(M) \subset M^{\prime}$ is $k_{0}$-nondegenerate if $k_{0}=\min \left\{k: H \in \mathcal{F}_{k}\right\}$ is a finite number.

Note that the definition of both $\mathcal{F}_{k_{0}}$ and the space of $k_{0}$-nondegenerate maps are independent of the choice of coordinates (see [13, Lemma 14]), hence these spaces are invariant under the action of $G$. Also notice that in the setup of Theorem 2 the space of 2-nondegenerate maps coincides with the set $\mathcal{F}_{2}$.

Our first main goal in this paper is to study the following property:

Definition 4. Let $M$ and $M^{\prime}$ be germs of submanifolds in $\mathbb{C}^{N}$ (resp. $\mathbb{C}^{N^{\prime}}$ ) around 0 , and let $H$ be a mapping of $M$ into $M^{\prime}$. We say that $H$ is locally rigid if $H$ projects to an isolated point in the quotient $\mathcal{H}\left(M, M^{\prime}\right) /_{G}$ of the space $\mathcal{H}\left(M, M^{\prime}\right)$ of holomorphic mappings from $M$ to $M^{\prime}$ with respect to the group of isotropies $G$. 
Remark 5. It is easy to show that $H \in \mathcal{H}\left(M, M^{\prime}\right)$ is locally rigid according to the definition above if and only if there exists a neighborhood $U$ of $H$ in $(\mathbb{C}\{Z\})^{N^{\prime}}$ such that for every $\hat{H} \in \mathcal{H}\left(M, M^{\prime}\right) \cap U$ there is $g \in G$ such that $\hat{H}=g H$. In other words, $H$ is locally rigid if and only if all the maps in $\mathcal{H}\left(M, M^{\prime}\right)$ which are close enough to $H$ are equivalent to $H$ (see Remark 12 in [7]).

\section{Jet parametrization}

In order to prove our main theorems we will show that in an appropriate sense, the infinitesimal deformations can be considered as a tangent space, by deducing a jet parametrization result for maps in $\mathcal{F}_{k}$ based on the work in [3, 12,13]. First we will introduce some notation.

Let $H: \mathbb{C}^{N} \rightarrow \mathbb{C}^{N^{\prime}}$ be a germ of a holomorphic map and let $k$ be an integer. We denote by $j_{0}^{k} H$ the $k$-jet of $H$ at 0 , that is the collection of all derivatives of order $\leq k$ of the components of $H$ at 0 . The space of all $k$-jets at 0 will be denoted by $J_{0}^{k}$ (we drop the dependence on $N$ and $N^{\prime}$, which will remain fixed, for better readability). We denote by $\Lambda$ coordinates in $J_{0}^{k}$ and write $\Lambda=\left(\Lambda^{\prime}, \Lambda_{N^{\prime}}\right)=\left(\Lambda_{1}, \ldots, \Lambda_{N^{\prime}-1}, \Lambda_{N^{\prime}}\right)$ with $\Lambda_{j}=\left(\Lambda_{j}^{\alpha, \beta}\right)$, where $\alpha \in \mathbb{N}_{0}^{n}, \beta \in \mathbb{N}_{0}^{d}$ and $0 \leq|\alpha|+|\beta| \leq k$. We have

$$
\Lambda=j_{0}^{k} H \text { if and only if } \Lambda_{j}^{\alpha, \beta}=\frac{1}{\alpha ! \beta !} \frac{\partial^{|\alpha|+|\beta|} H_{j}}{\partial z^{\alpha} \partial w^{\beta}}(0) .
$$

We can identify $k$-th order jets with polynomial maps of degree at most $k$ (taking $\mathbb{C}^{N}$ into $\mathbb{C}^{N^{\prime}}$ ) and will do so freely in the sequel. In particular, the composition of a jet with a jet is defined, as well as the composition of jets with other maps, provided that the source and target dimensions allow it.

We also need to recall the definition of certain subsets of $\mathbb{C}^{N}$, commonly referred to as the Segre sets. In order to do so we need to introduce some notation. For any $j \in \mathbb{N}$ let $\left(x_{1}, \ldots, x_{j}\right)\left(x_{\ell} \in \mathbb{C}^{n}\right)$ be coordinates for $\mathbb{C}^{n j}$. The Segre map of order $q \in \mathbb{N}$ is the map $S_{0}^{q}: \mathbb{C}^{n q} \rightarrow \mathbb{C}^{N}$ inductively defined as follows:

$$
S_{0}^{1}\left(x_{1}\right)=\left(x_{1}, 0\right), \quad S_{0}^{q}\left(x_{1}, \ldots, x_{q}\right)=\left(x_{1}, Q\left(x_{1}, \bar{S}_{0}^{q-1}\left(x_{2}, \ldots, x_{q}\right)\right)\right)
$$

where we denote by $\bar{S}_{0}^{q-1}$ the power series whose coefficients are conjugate to the ones of $S_{0}^{q-1}$ and $Q$ is a map as given in (1). In particular if $w-Q(z, \chi, \tau)=0$ is a local defining equation of the complexification of a $\mathrm{CR}$ submanifold $M \subset \mathbb{C}^{N}$, we say the Segre map $S_{0}^{q}$ is associated to $M$. The $q$-th Segre set $\mathcal{S}_{0}^{q} \subset \mathbb{C}^{N}$ is then the image of the map $S_{0}^{q}$. In what follows we will use the notation $x^{[j ; k]}=\left(x_{j}, \ldots, x_{k}\right)$. It is known from the Baouendi-Ebenfelt-Rothschild minimality criterion [1] that if $M$ is minimal at 0 , then $S_{0}^{j}$ is generically of full rank if $j$ is large enough. We recall that a germ of a CR submanifold $M \subset \mathbb{C}^{N}$ is called minimal at $p \in M$ if there is no germ of a CR submanifold $\tilde{M} \subsetneq M$ of $\mathbb{C}^{N}$ through $p$ having the same $\mathrm{CR}$ dimension as $M$ at $p$.

Theorem 6. Let $M \subset \mathbb{C}^{N}$ be the germ of a real-analytic, generic minimal submanifold, $0 \in$ $M$, and let $M^{\prime} \subset \mathbb{C}^{N^{\prime}}$ be a real-analytic generic submanifold germ. Let $k_{0} \in \mathbb{N}$ and $\mathbf{t} \leq d+1$ 
be the minimum integer, such that the Segre map $S_{0}^{\mathbf{t}}$ of order $\mathbf{t}$ associated to $M$ is generically of full rank. First suppose that $\mathbf{t}$ is even. There exists a finite collection of polynomials $q_{j}(\Lambda)$ on $J_{0}^{\mathrm{t} k_{0}}$ for $j \in J$, where $J$ is a suitable finite index set, open neighborhoods $\mathcal{U}_{j}$ of $\{0\} \times U_{j}$ in $\mathbb{C}^{N} \times J_{0}^{\mathrm{t} k_{0}}$, where $U_{j}=\left\{q_{j} \neq 0\right\}$ and holomorphic maps $\Phi_{j}: \mathcal{U}_{j} \rightarrow \mathbb{C}^{N^{\prime}}$ satisfying $\Phi_{j}(0, \Lambda)=0$, which are of the form

$$
\Phi_{j}(Z, \Lambda)=\sum_{\alpha \in \mathbb{N}_{0}^{N}} \frac{p_{j}^{\alpha}(\Lambda)}{q_{j}(\Lambda)^{d_{\alpha}^{j}}} Z^{\alpha}, \quad p_{j}^{\alpha}, q_{j} \in \mathbb{C}[\Lambda], \quad d_{\alpha}^{j} \in \mathbb{N}_{0},
$$

such that the following holds:

- For every $H \in \mathcal{F}_{k_{0}}$, in particular for every $k_{0}$-nondegenerate map $H$, there exists $j \in J$ such that $j_{0}^{\mathbf{t} k_{0}} H \in U_{j}$.

- For every $H \in \mathcal{F}_{k_{0}}$ with $j_{0}^{\mathrm{t} k_{0}} H \in U_{j}$ we have

$$
H(Z)=\Phi_{j}\left(Z, j_{0}^{\mathbf{t} k_{0}} H\right)
$$

In particular, there exist (real) polynomials $c_{k}^{j}, k \in \mathbb{N}$ on $J_{0}^{\mathrm{t} k_{0}}$ such that

$$
j_{0}^{\mathrm{t} k_{0}} \mathcal{F}_{k_{0}}=\bigcup_{j \in J}\left\{\Lambda \in J_{0}^{\mathrm{t} k_{0}}: q_{j}(\Lambda) \neq 0, c_{k}^{j}(\Lambda, \bar{\Lambda})=0\right\} .
$$

Analogous statements hold for $\mathbf{t}$ odd, where in this case all $p_{j}^{\alpha}$ and $q_{j}$ in the expansion of $\Phi_{j}$ in (4) depend antiholomorphic on $\Lambda$.

The proof of Theorem [6 will be split up into several lemmas. We define $K(t)=\mid\{\alpha \in$ $\left.\mathbb{N}_{0}^{N}:|\alpha| \leq t\right\} \mid$.

Lemma 7. Let $M$ and $M^{\prime}$ be as before. Fix multiindices $\left(\iota_{1}, \ldots, \iota_{N^{\prime}}\right)$ and integers $\ell^{1}, \ldots, \ell^{N^{\prime}}$ as above. Let $k_{0}=\max _{1 \leq m \leq N^{\prime}}\left|\iota_{m}\right|$. There is a holomorphic map $\Psi: \mathbb{C}^{N} \times \mathbb{C}^{N} \times \mathbb{C}^{K\left(k_{0}\right) N^{\prime}} \rightarrow$ $\mathbb{C}^{N^{\prime}}$ such that for every holomorphic map $H: \mathbb{C}^{N} \rightarrow \mathbb{C}^{N^{\prime}}$ satisfying (2) and $s(0) \neq 0$, where $s$ is given as in (3), we have

$$
H(Z)=\Psi\left(Z, \zeta, \partial^{k_{0}} \bar{H}(\zeta)\right)
$$

for $(Z, \zeta)$ in a neighborhood of 0 in $\mathcal{M}$, where $\partial^{k_{0}}$ denotes the collection of all derivatives up to order $k_{0}$. Furthermore there exist polynomials $P_{\alpha, \beta}, Q$ and integers $e_{\alpha, \beta}$ such that

$$
\Psi(Z, \zeta, W)=\sum_{\alpha, \beta \in \mathbb{N}_{0}^{N}} \frac{P_{\alpha, \beta}(W)}{Q^{e_{\alpha, \beta}(W)}} Z^{\alpha} \zeta^{\beta}
$$

In Lemma 7 the statement up until (66) is a reformulation of Prop. 25 in [13]. The expansion in (77) follows from the way the implicit function theorem is applied in the proof of Prop. 25, in a similar fashion as in [3, 12].

From now on all the jet parametrization mappings that will appear in the following lemmas will depend on the multiindices and integers fixed in Lemma 7 , For the sake of readability we will omit to write this dependence explicitly. 
Lemma 8. Under the assumptions of Lemma 7 the following holds: For all $\ell \in \mathbb{N}$ there exists a holomorphic mapping $\Psi_{\ell}: \mathbb{C}^{N} \times \mathbb{C}^{N} \times \mathbb{C}^{K\left(k_{0}+\ell\right) N^{\prime}} \rightarrow \mathbb{C}^{N^{\prime}}$ such that for every holomorphic map $H: \mathbb{C}^{N} \rightarrow \mathbb{C}^{N^{\prime}}$ satisfying (21) and $s(0) \neq 0$, where $s$ is given as in (3), we have

$$
\partial^{\ell} H(Z)=\Psi_{\ell}\left(Z, \zeta, \partial^{k_{0}+\ell} \bar{H}(\zeta)\right)
$$

for $(Z, \zeta)$ in a neighborhood of 0 in $\mathcal{M}$, where $\partial^{\ell}$ denotes the collection of all derivatives up to order $\ell$. Furthermore there exist polynomials $P_{\alpha, \beta}^{\ell}, Q_{\ell}$ and integers $e_{\alpha, \beta}^{\ell}$ such that

$$
\Psi_{\ell}(Z, \zeta, W)=\sum_{\alpha, \beta \in \mathbb{N}_{0}^{N}} \frac{P_{\alpha, \beta}^{\ell}(W)}{Q_{\ell}^{e_{\alpha, \beta}^{\ell}}(W)} Z^{\alpha} \zeta^{\beta} .
$$

Lemma 8 follows by differentiating (6) along the vector fields $S$ and $T$ introduced above, see Cor. 26 of [13].

The next step is to evaluate (6) along the Segre sets.

Corollary 9. Under the assumptions of Lemma 7 the following holds: For fixed $q \in \mathbb{N}$ there exists a holomorphic mapping $\varphi_{q}: \mathbb{C}^{q n} \times \mathbb{C}^{K\left(q k_{0}\right) N^{\prime}} \rightarrow \mathbb{C}^{N^{\prime}}$ such that for every holomorphic map $H: \mathbb{C}^{N} \rightarrow \mathbb{C}^{N^{\prime}}$ satisfying (2) and $s(0) \neq 0$, where $s$ is given as in (3), we have

$$
H\left(S_{0}^{q}\left(x^{[1 ; q]}\right)\right)=\varphi_{q}\left(x^{[1 ; q]}, j_{0}^{q} H\right) .
$$

Furthermore there exist (holomorphic) polynomials $R_{\gamma}^{q}, S_{q}$ and integers $m_{\gamma}^{q}$ such that

$$
\varphi_{q}\left(x^{[1 ; q]}, \Lambda\right)= \begin{cases}\sum_{\gamma \in \mathbb{N}_{0}^{q n}} \frac{R_{\gamma}^{q}(\Lambda)}{S_{q}^{m_{\gamma}^{q}}(\Lambda)}\left(x^{[1 ; q]}\right)^{\gamma} \quad q \text { even } \\ \sum_{\gamma \in \mathbb{N}_{0}^{q n}} \frac{R_{\gamma}^{q}(\bar{\Lambda})}{S_{q}^{m_{\gamma}^{q}}(\bar{\Lambda})}\left(x^{[1 ; q]}\right)^{\gamma} \quad q \text { odd } .\end{cases}
$$

Proof. Fix $q \in \mathbb{N}$. We begin by putting $Z=S_{0}^{q}\left(x^{[1 ; q]}\right)$ and $\zeta=\bar{S}_{0}^{q-1}\left(x^{[2 ; q]}\right)$, so that $(Z, \zeta) \in \mathcal{M}$, in the identity (6), in order to obtain

$$
H\left(S_{0}^{q}\left(x^{[1 ; q]}\right)\right)=\Psi\left(S_{0}^{q}\left(x^{[1 ; q]}\right), \bar{S}_{0}^{q-1}\left(x^{[2 ; q]}\right), \partial^{k_{0}} \bar{H}\left(\bar{S}_{0}^{q-1}\left(x^{[2 ; q]}\right)\right)\right) .
$$

This equation means that one can determine the value of any solution of (2), at least along $\mathcal{S}_{0}^{q}$, by knowing the values of its derivatives along $\mathcal{S}_{0}^{q-1}$. To determine the latter, we put $\zeta=\bar{S}_{0}^{q-1}\left(x^{[2 ; q]}\right)$ and $Z=S_{0}^{q-2}\left(x^{[3 ; q]}\right)$ (again so that $(Z, \zeta) \in \mathcal{M}$ ) in the conjugate of (8)):

$$
\partial^{\ell} \bar{H}\left(\bar{S}_{0}^{q-1}\left(x^{[2 ; q]}\right)\right)=\bar{\Psi}_{\ell}\left(\bar{S}_{0}^{q-1}\left(x^{[2 ; q]}\right), S_{0}^{q-2}\left(x^{[3 ; q]}\right), \partial^{k_{0}+\ell} H\left(S_{0}^{q-2}\left(x^{[3 ; q]}\right)\right)\right) .
$$

By substituting (13) for $\ell=k_{0}$ into (12), we get that the values of $H$ along $\mathcal{S}_{0}^{q}$ are determined by the values of their $2 k_{0}$-th order jet along $\mathcal{S}_{0}^{q-2}$. Iterating this argument $q$ times we prove (10). To show (11) we use (77) and (9) at every step; the desired expansion follows by a (cumbersome but) straightforward computation. In particular one derives that $j_{0}^{q k_{0}} H \in\left\{S_{q} \neq 0\right\}$, whenever $s(0) \neq 0$. For more details see [3, 12] 
Proof of Theorem [6. By the choice of $\mathbf{t} \leq d+1$, the Segre map $S_{0}^{\mathbf{t}}$ is generically of maximal rank, and we can therefore define the finite number $\nu\left(S_{0}^{\mathbf{t}}\right)$ as the minimum order of vanishing of minor of maximal size of the Jacobian of $S_{0}^{\mathbf{t}}$.

We can thus appeal to Theorem 5 from [12] and obtain that there exist a neighborhood $\mathcal{V}$ of $S_{0}^{\mathbf{t}}$ in $\left(\mathbb{C}\left\{x^{[1 ; \mathbf{t}]}\right\}\right)^{N}$ and a holomorphic map

$$
\phi: \mathcal{V} \times \mathbb{C}\left\{x^{[1 ; \mathbf{t}]}\right\} \rightarrow \mathbb{C}\{Z\}
$$

such that $\phi(A, h \circ A)=h$ for all $A \in \mathcal{V}$ with $\nu(A)=\nu\left(S_{0}^{\mathbf{t}}\right)$, and for all $h \in \mathbb{C}\{Z\}$.

Now, define $J$ as the set of all the sequences of multiindices $\left(\iota_{1}, \ldots, \iota_{N^{\prime}}\right)$ and integers $\ell^{1}, \ldots, \ell^{N^{\prime}}$ as in Lemma 7 with $k_{0}=\max _{1 \leq m \leq N^{\prime}}\left|\iota_{m}\right|$. For any $j \in J$, Corollary 9 with $q=\mathbf{t}$ provides the existence of a map $\varphi_{\mathbf{t}}=\varphi_{\mathbf{t}, j}$ satisfying (10). We set $\Phi_{j}(\cdot, \Lambda)=\phi\left(S_{0}^{\mathbf{t}}, \varphi_{\mathbf{t}, j}(\cdot, \Lambda)\right)$ so that by the properties of $\varphi_{\mathbf{t}, j}$ and $\phi$ the map $\Phi_{j}$ depends holomorphically on $\Lambda=j_{0}^{\mathrm{t}} H$ (or $\bar{\Lambda}$, respectively, if $j$ is odd). By setting $q_{j}(\Lambda, \bar{\Lambda})=S_{\mathbf{t}, j}(\Lambda)$, where $S_{\mathbf{t}, j}$ is given in (11), a direct computation using (11) and Thm. 5 in [12] (more precisely (42) of Thm. 6 of [12]), allows to derive the expansion in (4).

It follows from Corollary 9 and Thm. 5 in [12] that $H(Z)=\Phi_{j}\left(Z, j_{0}^{\text {t } k_{0}} H\right)$ whenever $H$ is a solution of (2) and $s(0) \neq 0$, where $s$ is given as in (3) with the sequence of multiindices corresponding to $j$. In particular if $H$ is $k_{0}$-nondegenerate by definition there exists $j \in J$ such that $s(0) \neq 0$, which by the arguments above is equivalent to the condition $j_{0}^{\mathrm{t} k_{0}} H \in U_{j}$.

Finally, the remaining statement can be proved by setting $H(Z)=\Phi_{j}(Z, \Lambda)$ in (2) and expanding it as a power series in $(z, \chi, \tau)$ : the coefficients of this power series depend polynomially on $\Lambda, \bar{\Lambda}$, so that the defining equations (5) can be obtained by setting all the coefficients to 0 .

\section{INFINITESIMAL DEFORMATIONS}

In the following we refer to the notation of Theorem 6. For any $j \in J$ let $A_{j} \subset U_{j}$ be the real-analytic set defined as

$$
A_{j}=\left\{\Lambda \in U_{j}: c_{k}^{j}(\Lambda, \bar{\Lambda})=0, k \in \mathbb{N}\right\} .
$$

By Theorem 6 putting $A:=\bigcup_{j} A_{j}$ we have $j_{0}^{\mathrm{t} k_{0}}\left(\mathcal{F}_{k_{0}}\right)=A$; in particular $A$ contains the set of $\mathbf{t} k_{0}$-jets of all $k_{0}$-nondegenerate mappings of $M$ into $M^{\prime}$. In fact we can say more:

Lemma 10. For every $j \in J$ we define $\mathcal{F}_{k_{0}, j}:=\mathcal{F}_{k_{0}} \cap\left(j_{0}^{\mathrm{t} k_{0}}\right)^{-1}\left(U_{j}\right)$. The map $\Phi_{j}: A_{j} \rightarrow$ $\mathcal{F}_{k_{0}, j}$ is a homeomorphism.

This result is proved exactly as Lemma 19 in [7] as a direct consequence of Theorem [6. For each $j \in J$ the restriction of $\Phi_{j}$ to $\mathcal{U}_{j} \cap\left(\mathbb{C}^{N} \times A_{j}\right)$ gives rise to a map

$$
A_{j} \ni \Lambda \rightarrow \Phi_{j}(\Lambda) \in(\mathbb{C}\{Z\})^{N^{\prime}}, \Phi_{j}(\Lambda)(Z)=\Phi_{j}(Z, \Lambda)
$$

from $A_{j}$ to the space $(\mathbb{C}\{Z\})^{N^{\prime}}$.

Let $X \subset A_{j}$ be any regular (real-analytic) submanifold, and fix $\Lambda_{0} \in X$. In what follows we focus on $\left.\Phi_{j}\right|_{X}$. Note that if we restrict to a small enough neighborhood of $\Lambda_{0}$ in $X$ (which we again denote by $X$ ) the maps $\Phi_{j}(\Lambda)$ for all $\Lambda \in X$ all have a common radius of 
convergence $R$, so that we can consider the restriction of $\Phi_{j}$ to $X$ as a map valued in the Banach space $\operatorname{Hol}\left(\overline{B_{R}(0)}, \mathbb{C}^{N^{\prime}}\right)$, the space of holomorphic mappings from $B_{R}(0)$ to $\mathbb{C}^{N^{\prime}}$, which are continuous up to $\overline{B_{R}(0)}$, where $B_{R}(0)$ denotes the ball with radius $R>0$ in $\mathbb{C}^{N}$.

We also remark that the map $\Phi_{j}: X \rightarrow(\mathbb{C}\{Z\})^{N^{\prime}}$ is of class $C^{\infty}$. We consider its Fréchet derivative $D \Phi_{j}\left(\Lambda_{0}\right)$ at $\Lambda_{0}$ as a map $T_{\Lambda_{0}} X \rightarrow T_{\Phi_{j}\left(\Lambda_{0}\right)}(\mathbb{C}\{Z\})^{N^{\prime}} \cong(\mathbb{C}\{Z\})^{N^{\prime}}$.

We will need a special subspace of $T_{\Phi_{j}\left(\Lambda_{0}\right)}(\mathbb{C}\{Z\})^{N^{\prime}}$. The following definition, already stated in Section 1, was first given in [5], see also [7].

Definition 11. Let $M$ and $M^{\prime}$ be as above and $H:\left(\mathbb{C}^{N}, 0\right) \rightarrow\left(\mathbb{C}^{N^{\prime}}, 0\right)$ a map with $H(M) \subset M^{\prime}$. Then a vector

$$
V=\sum_{j=1}^{N^{\prime}} \alpha_{j}(Z) \frac{\partial}{\partial Z_{j}^{\prime}} \in T_{H}(\mathbb{C}\{Z\})^{N^{\prime}}
$$

is an infinitesimal deformation of $H$ if the real part of $V$ is tangent to $M^{\prime}$ along $H(M)$, i.e. if for one (and hence every) defining function $\rho^{\prime}=\left(\rho_{1}^{\prime}, \ldots, \rho_{d^{\prime}}^{\prime}\right)$ of $M^{\prime}$, the components of $V$ satisfy the following linear system

$$
\operatorname{Re}\left(\sum_{j=1}^{N^{\prime}} \alpha_{j}(Z) \frac{\partial \rho_{\ell}^{\prime}}{\partial Z_{j}^{\prime}}(H(Z), \overline{H(Z)})\right)=0 \text { for } Z \in M, \ell=1, \ldots, d^{\prime} .
$$

We denote this subspace of $T_{H}(\mathbb{C}\{Z\})^{N^{\prime}}$ by $\mathfrak{h o l}_{0}(H)$.

With the same proof as in [7] we derive the following property, which motivates the definition above:

Lemma 12. The image of $T_{\Lambda_{0}} X$ by $D \Phi_{j}\left(\Lambda_{0}\right)$ is contained in $\mathfrak{h o l}_{0}\left(\Phi_{j}\left(\Lambda_{0}\right)\right)$.

The next lemmas give some properties of infinitesimal deformations that will be needed in section 6 to give the proofs our main theorems. The first lemma comes from the jet parametrization for solutions of (15) obtained in section 5 in [7]. Its proof is precisely the one of Cor. 32 in [7] using Prop. 29 instead of Prop. 31 and keeping $Q$ fixed.

Lemma 13. Fix $M$ and $M^{\prime}$ given as above. For any $H \in \mathcal{F}_{k_{0}}$, the dimension $\operatorname{dim}_{\mathbb{R}}\left(\mathfrak{h o l}_{0}(H)\right)$ of the space of infinitesimal deformations of $H$ is finite. Moreover, the function $\operatorname{dim}_{\mathbb{R}}\left(\mathfrak{h o l}_{0}(\cdot)\right)$ : $\mathcal{F}_{k_{0}} \rightarrow \mathbb{N}_{0}$ is upper semicontinuous, i.e. for any $H \in \mathcal{F}_{k_{0}}$ there exists a neighborhood $\mathcal{V}$ of $H$ in $\mathcal{F}_{k_{0}}$ such that for any $H^{\prime} \in \mathcal{V}$ we have $\operatorname{dim}_{\mathbb{R}}\left(\mathfrak{h o l}_{0}\left(H^{\prime}\right)\right) \leq \operatorname{dim}_{\mathbb{R}}\left(\mathfrak{h o l}_{0}(H)\right)$.

The following lemma follows from Theorem 6, Lemma 12 and Lemma 13 with the same proof as Lemma 23 in [7].

Lemma 14. Let $\Lambda_{0} \in A_{j}$, and suppose that $\operatorname{dim}_{\mathbb{R}} \mathfrak{h o l}_{0}\left(\Phi_{j}\left(\Lambda_{0}\right)\right)=\ell$. Then there exists a neighborhood $U$ of $\Lambda_{0}$ in $J_{0}^{\mathrm{t} k_{0}}$ such that, if $X \subset A_{j}$ is a submanifold such that $X \cap U \neq \emptyset$, then $\operatorname{dim}_{\mathbb{R}}(X) \leq \ell$. 


\section{Properties of the Group ACtion}

In this section we deduce some results which will be used to prove Theorem 2, Thus we consider strictly pseudoconvex hypersurfaces $M \subset \mathbb{C}^{N}$ and $M^{\prime} \subset \mathbb{C}^{N^{\prime}}$. In the coordinates introduced in section 2 this means that the CR-dimension of $M$ and $M^{\prime}$ are equal to $n=N-1$ and $n^{\prime}=N^{\prime}-1$ respectively (and $d=d^{\prime}=1$ ).

More specifically we are interested in describing some properties of the action of the isotropy group on 2-nondegenerate embeddings, or more precisely on the set of their 4-jets (which by Theorem 6 parametrize $\mathcal{F}_{2}$ ). To this end we first give a brief description of the isotropy groups of the spheres $\mathbb{H}^{n+1}=\left\{(z, w) \in \mathbb{C}^{n+1}: \operatorname{Im} w=\|z\|^{2}\right\}$. Let $\Gamma_{n}=$ $\mathbb{R}^{+} \times \mathbb{R} \times U(n) \times \mathbb{C}^{n}$ be a parameter space. Then the map

$$
\Gamma_{n} \ni \gamma=(\lambda, r, U, c) \rightarrow \sigma_{\gamma}(z, w)=\frac{\left(\lambda U^{t}(z+c w), \lambda^{2} w\right)}{1-2 i\langle\bar{c}, z\rangle+\left(r-i\|c\|^{2}\right) w} \in \operatorname{Aut}_{0}\left(\mathbb{H}^{n+1}\right)
$$

is a diffeomorphism between $\Gamma_{n}$ and $\operatorname{Aut}_{0}\left(\mathbb{H}^{n+1}\right)$ : here we denote by $\langle\cdot, \cdot\rangle$ the product on $\mathbb{C}^{n}$ given by $\langle z, \widetilde{z}\rangle=\sum_{j=1}^{n} z_{j} \widetilde{z}_{j}$ and we write $\|z\|^{2}=\langle\bar{z}, z\rangle$.

The first property we are going to study is properness: we remind the reader that the action of a topological group $\mathcal{G}$ on a space $X$ is said to be proper if the map $\mathcal{G} \times X \rightarrow X \times X$ given by $(g, x) \mapsto(x, g x)$ is proper.

We will actually prove properness of the action on a particular subset of $J_{0}^{4}$ : let $E$ be the subset of $J_{0}^{4}$ defined by

$$
E=\left\{\Lambda_{N^{\prime}}^{\alpha, 0}=0,|\alpha| \leq 2,0 \neq \Lambda_{N^{\prime}}^{0,1}=\left\|\Lambda^{\prime \beta, 0}\right\|^{2},|\beta|=1,\left\langle\bar{\Lambda}^{\prime \gamma, 0}, \Lambda^{\prime \delta, 0}\right\rangle=0, \gamma \neq \delta,|\gamma|=|\delta|=1\right\} .
$$

One can verify in a straightforward manner the following properties of $E$ :

- $E$ is a (real algebraic) submanifold of $J_{0}^{4}$.

- For $M=\left\{\operatorname{Im} w=\|z\|^{2}+O(2)\right\}$ and $M^{\prime}=\left\{\operatorname{Im} w^{\prime}=\left\|z^{\prime}\right\|^{2}+O(2)\right\}$ the set $E$ contains the 4-th jet of any non-constant map from $M$ to $M^{\prime}$.

- $E$ is invariant under the action of $\operatorname{Aut}_{0}\left(\mathbb{H}^{N}\right) \times \operatorname{Aut}_{0}\left(\mathbb{H}^{N^{\prime}}\right)$, cf. Lemma 14 in [7].

Lemma 15. Suppose that $M \nsubseteq \mathbb{H}^{N}$ is strictly pseudoconvex and $M^{\prime}=\mathbb{H}^{N^{\prime}}$. Then the action of $G=\operatorname{Aut}_{0}(M) \times \operatorname{Aut}_{0}\left(\mathbb{H}^{N^{\prime}}\right)$ on $E$ is proper.

Proof. The proof follows closely the one of [7, Lemma 15]. With the same argument as there using the compactness of $\operatorname{Aut}_{0}(M)$, which follows from the assumption that $M ¥ \mathbb{H}^{N}$ (see [4]), we can reduce to showing the following: let $C>1$, and $\left\{\left(\Lambda_{m}, \widetilde{\Lambda}_{m}\right)\right\}_{m \in \mathbb{N}} \subset E \times E$, $\left(\sigma_{m}^{\prime}\right)_{m \in \mathbb{N}} \subset \operatorname{Aut}_{0}\left(\mathbb{H}^{N^{\prime}}\right)$ be sequences such that $\left|\Lambda_{m}\right|,\left|\widetilde{\Lambda}_{m}\right| \leq C,\left|\left(\Lambda_{m}\right)_{N^{\prime}}^{0,1}\right|,\left|\left(\widetilde{\Lambda}_{m}\right)_{N^{\prime}}^{0,1}\right| \geq 1 / C$ and

$$
\widetilde{\Lambda}_{m}=\sigma_{m}^{\prime} \circ \Lambda_{m}
$$

for all $m \in \mathbb{N}$. Then $\sigma_{m}^{\prime}$ admits a convergent subsequence.

Using the parametrization (16), this amounts to showing that the preimage $\left\{\gamma_{m}^{\prime}=\right.$ $\left.\left(\lambda_{m}^{\prime}, r_{m}^{\prime}, U_{m}^{\prime}, c_{m}^{\prime}\right)\right\}$ of $\sigma_{m}^{\prime}$ in the parameter space $\Gamma^{\prime}$ is relatively compact, that is $\left|r_{m}^{\prime}\right|,\left\|c_{m}^{\prime}\right\|, \lambda_{m}^{\prime}$ and $1 / \lambda_{m}^{\prime}$ are bounded, since $U\left(N^{\prime}-1\right)$ is a compact group. 
Looking at the $N^{\prime}$-th component of the first jet of (17), we get

$$
\lambda_{m}^{\prime 2}\left(\Lambda_{m}\right)_{N^{\prime}}^{0,1}=\left(\widetilde{\Lambda}_{m}\right)_{N^{\prime}}^{0,1}
$$

and hence $\lambda_{m}^{\prime}$ and $1 / \lambda_{m}^{\prime}$ are bounded.

Considering the first $N^{\prime}-1$ components of the first jet of (17) we obtain

$$
\lambda_{m}^{\prime} U_{m}^{\prime} \Lambda_{m}^{\prime 0,1}+\left(\Lambda_{m}\right)_{N^{\prime}}^{0,1} c_{m}^{\prime}=\widetilde{\Lambda}_{m}^{\prime 0,1}
$$

hence

$$
\left\|c_{m}^{\prime}\right\| \leq \frac{1}{\left|\left(\Lambda_{m}\right)_{N^{\prime}}^{0,1}\right|}\left\|\widetilde{\Lambda}_{m}^{\prime 0,1}-\lambda_{m}^{\prime} U_{m}^{\prime} \Lambda_{m}^{\prime 0,1}\right\|
$$

which implies that $c_{m}^{\prime}$ is bounded in $\mathbb{C}^{N^{\prime}-1}$. Finally, we consider the $N^{\prime}$-th component of the second jet of (17), which gives us

$$
\left(\widetilde{\Lambda}_{m}\right)_{N^{\prime}}^{0,2}=-2 \lambda_{m}^{\prime 2}\left(\left(\Lambda_{m}\right)_{N^{\prime}}^{0,1}\right)^{2} r_{m}^{\prime}+R_{m}
$$

where $R_{m}$ is a polynomial expression in the second jet of $\Lambda_{m}$, in $\lambda_{m}^{\prime}$ and in the coefficients of $c_{m}^{\prime}$ and $U_{m}^{\prime}$ (but which does not depend on $r_{m}^{\prime}$ ). This shows that the sequence $r_{m}^{\prime}$ is bounded in $\mathbb{R}$, and concludes the proof.

Next, we consider the case $M=\mathbb{H}^{N}$ and $M^{\prime} ¥ \mathbb{H}^{N^{\prime}}$. The proof is quite similar to the previous one, but does not really reduce to it.

Lemma 16. The action of $\operatorname{Aut}_{0}\left(\mathbb{H}^{N}\right) \times \operatorname{Aut}_{0}\left(M^{\prime}\right)$ on $E$ is proper.

Proof. By the compactness of $\operatorname{Aut}_{0}\left(M^{\prime}\right)$ as in the previous lemma it is enough to show the following: let $C>1$, and let $\left\{\left(\Lambda_{m}, \widetilde{\Lambda}_{m}\right)\right\}_{m \in \mathbb{N}} \subset E \times E,\left(\sigma_{m}\right)_{m \in \mathbb{N}} \subset \operatorname{Aut}_{0}\left(\mathbb{H}^{N}\right)$ be sequences such that $\left|\Lambda_{m}\right|,\left|\widetilde{\Lambda}_{m}\right| \leq C,\left|\left(\Lambda_{m}\right)_{N^{\prime}}^{0,1}\right|,\left|\left(\widetilde{\Lambda}_{m}\right)_{N^{\prime}}^{0,1}\right| \geq 1 / C$ and

$$
\widetilde{\Lambda}_{m}=\Lambda_{m} \circ \sigma_{m}
$$

for all $m \in \mathbb{N}$. Then $\sigma_{m}$ admits a convergent subsequence.

The $N^{\prime}$-th component of the first jet of (18) gives

$$
\lambda_{m}^{2}\left(\Lambda_{m}\right)_{N^{\prime}}^{0,1}=\left(\widetilde{\Lambda}_{m}\right)_{N^{\prime}}^{0,1},
$$

which implies that the sequence $\lambda_{m}$ is bounded above and below.

Given $\Lambda \in J_{0}^{4}$ we denote by $\Lambda^{\prime 1,0}$ the $\left(N^{\prime}-1\right) \times(N-1)$-matrix given by $\left(\Lambda_{j}^{\prime \alpha, 0}\right), j=$ $1, \ldots, N^{\prime}-1, \alpha \in \mathbb{N}_{0}^{N-1}$ with $|\alpha|=1$. The first $N^{\prime}-1$ components of (the $(0,1)$-part of) the first jet of (18) can then be written as follows:

$$
\lambda_{m}\left(\lambda_{m} \Lambda_{m}^{\prime 0,1}+\Lambda_{m}^{\prime 1,0} U_{m} c_{m}\right)=\widetilde{\Lambda}_{m}^{\prime 0,1},
$$

therefore

$$
\frac{1}{\lambda_{m}} \widetilde{\Lambda}_{m}^{\prime 0,1}-\lambda_{m} \Lambda_{m}^{\prime 0,1}=\Lambda_{m}^{\prime 1,0} U_{m} c_{m}
$$


By definition of $E$ we can write the matrix $\Lambda_{m}^{\prime 1,0}$ as $\sqrt{\left(\Lambda_{m}\right)_{N^{\prime}}^{0,1}} A_{m}$, where $A_{m}$ is a semiunitary matrix, i.e. ${ }^{t} \bar{A}_{m} A_{m}=I_{N-1}$, thus we have

$$
\left\|\Lambda_{m}^{\prime 1,0} U_{m} c_{m}\right\|^{2}=\left(\Lambda_{m}\right)_{N^{\prime}}^{0,1}\left\|A_{m} U_{m} c_{m}\right\|^{2}=\left(\Lambda_{m}\right)_{N^{\prime}}^{0,1}\left\|U_{m} c_{m}\right\|^{2}=\left(\Lambda_{m}\right)_{N^{\prime}}^{0,1}\left\|c_{m}\right\|^{2},
$$

so that by the estimate on $\left(\Lambda_{m}\right)_{N^{\prime}}^{0,1}$ it holds that

$$
\frac{\left\|c_{m}\right\|}{\sqrt{C}} \leq \lambda_{m}\left\|\Lambda_{m}^{\prime 0,1}\right\|+\frac{1}{\lambda_{m}}\left\|\widetilde{\Lambda}_{m}^{\prime 0,1}\right\|
$$

which implies the boundedness of $c_{m}$ in $\mathbb{C}^{N-1}$. Finally, we consider the $N^{\prime}$-th component of the second jet of (18), which gives the equation

$$
\left(\widetilde{\Lambda}_{m}\right)_{N^{\prime}}^{0,2}=-\lambda_{m}^{2}\left(\Lambda_{m}\right)_{N^{\prime}}^{0,1} r_{m}+R_{m}
$$

where $R_{m}$ is a polynomial expression in the second jet of $\Lambda_{m}$ and in $\lambda_{m}, c_{m}, U_{m}$, not depending on $r_{m}$. This implies that the sequence $r_{m}$ is bounded in $\mathbb{R}$, and concludes the proof.

Next we are going to prove the freeness of the action of the isotropy group of the target manifold. In order to do so, we first introduce for any fixed map $H$, in a way similar to Lemma 17 in [7], coordinates such that

- the map $H$ is of the form $(z, F(z, w), w)$ for a certain germ of holomorphic function $F: \mathbb{C}^{N} \rightarrow \mathbb{C}^{\ell}$, where $\ell=N^{\prime}-N$, such that $F(0)=0$;

- the automorphism group of $M^{\prime}$ at 0 is a subgroup of $\operatorname{Aut}_{0}\left(\mathbb{H}^{N^{\prime}}\right)$.

Lemma 17. Let $\Lambda \in E$ be the 4-jet of a map of the form $(z, w) \rightarrow(z, F(z, w), w) \in \mathcal{F}_{2}$. Then the stabilizer of $\Lambda$ under the action of $G^{\prime}=\{\mathrm{id}\} \times \operatorname{Aut}_{0}\left(\mathbb{H}^{N^{\prime}}\right)$ is trivial.

Proof. For $v \in \mathbb{C}^{m}$ we denote by $v_{[j ; k]}$ the coordinates $\left(v_{j}, \ldots, v_{k}\right)$ for $1 \leq j \leq k \leq m$. First using that $\Lambda \in E$ we deduce $\Lambda_{N}^{\alpha, 0}=\cdots=\Lambda_{N^{\prime}-1}^{\alpha, 0}=0$ for all $\alpha$ such that $|\alpha|=1$. Indeed for all $\alpha$ with $|\alpha|=1$ we have

$$
1=\Lambda_{N^{\prime}}^{0,1}=\left\|\Lambda^{\prime \alpha, 0}\right\|^{2}=\sum_{j=1}^{N-1}\left|\Lambda_{j}^{\alpha, 0}\right|^{2}+\sum_{j=N}^{N^{\prime}-1}\left|\Lambda_{j}^{\alpha, 0}\right|^{2}=1+\sum_{j=N}^{N^{\prime}-1}\left|\Lambda_{j}^{\alpha, 0}\right|^{2},
$$

since for every $\alpha$ there exists exactly one $j_{\alpha}$, such that $\Lambda_{j_{\alpha}}^{\alpha, 0}=1$ and $\Lambda_{j}^{\alpha, 0}=0$ for all $j \neq j_{\alpha}, 1 \leq j \leq N-1$ (by the form of $\Lambda$ ). In other words the entries of the last $\ell$ rows of the $(N-1+\ell) \times(N-1)$ matrix $\Lambda^{\prime 1,0}$ defined in the proof of Lemma 16 are all zeros, while the rest of the matrix is an $(N-1) \times(N-1)$ identity matrix.

Let $\Lambda$ be as in the assumptions and $\sigma^{\prime} \in G^{\prime}$ in the stabilizer of $\Lambda$, that is

$$
\Lambda=\sigma^{\prime} \circ \Lambda \text {. }
$$

Let $\sigma^{\prime}$ be parametrized by $\gamma^{\prime}=\left(\lambda^{\prime}, r^{\prime}, U^{\prime}, c^{\prime}\right) \in \Gamma^{\prime}$. We will show that $\sigma^{\prime}=$ id by following similar computations analogous to the ones in Lemma 15. Looking at the $N^{\prime}$-th component of the first jet of (19) we see that $\left(\lambda^{\prime}\right)^{2}=1$, hence $\lambda^{\prime}=1$ since $\lambda^{\prime} \in \mathbb{R}^{+}$. 
Considering the first $N^{\prime}-1$ components of the first jet of (19), we get $U^{\prime} \Lambda^{\prime 1,0}=\Lambda^{\prime 1,0}$, hence $U^{\prime}$ is a block diagonal matrix with first block being $I_{N-1}$ and the second block a $\ell \times \ell$ unitary matrix $U^{\prime \prime}$. Furthermore, we obtain

$$
U^{\prime}{ }^{t}\left(0, \ldots, 0, \Lambda_{\left[N ; N^{\prime}-1\right]}^{0,1}\right)+c^{\prime}={ }^{t}\left(0, \ldots, 0, \Lambda_{\left[N ; N^{\prime}-1\right]}^{0,1}\right),
$$

i.e. $c_{j}^{\prime}=0$ for $1 \leq j \leq N-1$ and $c_{\left[N ; N^{\prime}-1\right]}^{\prime}=\left(I_{\ell}-U^{\prime \prime}\right) \Lambda_{\left[N ; N^{\prime}-1\right]}^{0,1}$.

Since $\Lambda$ comes from a map in $\mathcal{F}_{2}$ it follows that there exists a collection of multiindices $\Delta=\left(\delta_{1}, \ldots, \delta_{\ell}\right)$ with $\left|\delta_{j}\right|=2$ such that the $\ell \times \ell$-matrix $\Lambda_{\left[N ; N^{\prime}-1\right]}^{\Delta, 0}$ (whose $(j, k)$-entry is $\left.\Lambda_{j+N-1}^{\delta_{k}, 0}\right)$ is invertible. Indeed in the Definition 3 we can take the sequence of multiindices $\left(\iota_{1}, \ldots, \iota_{N^{\prime}}\right)$ to be $\iota_{1}=0$, for $2 \leq k \leq N$ the multiindex $\iota_{k}=(0, \ldots, 0,1,0, \ldots, 0)$ (where the 1 appears in the $k-1$-th entry) and $\iota_{N+j}=\delta_{j}$ for $1 \leq j \leq \ell$. With this choice the determinant $s$ at 0 of the matrix in Definition 3 is

$$
s(0)=\operatorname{det}\left(\begin{array}{cccccc}
0 & 0 & \cdots & 0 & 0 & 1 \\
1 & 0 & \cdots & 0 & 0 & 0 \\
0 & 1 & \cdots & 0 & 0 & 0 \\
\vdots & \vdots & \ddots & \vdots & \vdots & \vdots \\
0 & 0 & \cdots & 1 & 0 & 0 \\
\Lambda_{1}^{\Delta, 0} & \Lambda_{2}^{\Delta, 0} & \cdots & \Lambda_{N-1}^{\Delta, 0} & \Lambda_{\left[N ; N^{\prime}-1\right]}^{\Delta, 0} & 0
\end{array}\right)= \pm \operatorname{det} \Lambda_{\left[N ; N^{\prime}-1\right]}^{\Delta, 0},
$$

so that $\operatorname{det} \Lambda_{\left[N ; N^{\prime}-1\right]}^{\Delta, 0} \neq 0$. Using this fact and considering the $\left[N ; N^{\prime}-1\right]$ components of the second jet of (19) we have:

$$
U^{\prime \prime} \Lambda_{\left[N ; N^{\prime}-1\right]}^{\Delta}=\Lambda_{\left[N ; N^{\prime}-1\right]}^{\Delta, 0} \text {, i.e. } U^{\prime \prime}=I_{\ell}, c_{\left[N ; N^{\prime}-1\right]}^{\prime}=0,
$$

since $\Lambda_{\left[N ; N^{\prime}-1\right]}^{\Delta}$ is invertible. Finally taking into account the previous computations the remaining equation in the 2 -jet of the $N^{\prime}$-th component in (19) becomes

$$
-r^{\prime}=\Lambda_{N^{\prime}}^{0,2}=0
$$

which shows that $\sigma^{\prime}=\mathrm{id}$.

\section{Proofs of the MAIN Results}

In this section we are going to prove Theorems 1 and 2 ,

Theorem 18. Let $M, M^{\prime}$ be as in Theorem [6, let $A$ be defined as in (14), $\Lambda_{0} \in A$, and let $j \in J$ be such that $\Lambda_{0} \in A_{j}$. Suppose that $\operatorname{dim}_{\mathbb{R}} \mathfrak{h o l} \mathfrak{l}_{0}\left(\Phi_{j}\left(\Lambda_{0}\right)\right)=0$, then $\Phi_{j}\left(\Lambda_{0}\right)$ is isolated in $\mathcal{F}_{k_{0}}$.

Proof. By Lemma 14, there is a neighborhood $U$ of $\Lambda_{0}$ in $J_{0}^{\mathrm{t} k_{0}}$ such that $U \cap A_{j}$ does not contain any manifold of positive dimension. It follows that $U \cap A_{j}$ is a discrete set: by Lemma 10 we have that $\Phi_{j}\left(\Lambda_{0}\right)$ is in turn isolated in $\mathcal{F}_{k_{0}}$.

Now we have all the ingredients to give a proof of Theorem 1: 
Proof of Theorem 1. Let $H$ be a finitely nondegenerate map. Then there exists an integer $k_{0}$ such that $H \in \mathcal{F}_{k_{0}}$ (see Definition [3). Set $\Lambda_{0}=j_{0}^{\text {t } k_{0}} H$, then there exists $j \in J$, such that $\Lambda_{0} \in A_{j}$ and $H=\Phi_{j}\left(\Lambda_{0}\right)$. By Theorem $18 H$ is isolated in $\mathcal{F}_{k_{0}}$, but since $\mathcal{F}_{k_{0}}$ is an open set of $\mathcal{H}\left(M, M^{\prime}\right)$ (see again Definition 3), it follows that $H$ is isolated in $\mathcal{H}\left(M, M^{\prime}\right)$. In particular $H$ is locally rigid by Remark 5 .

Let us now turn to Theorem [2, In the setting the jet space in Theorem 6 can be taken to be $J_{0}^{4}$, since $k_{0}=2$ and $\mathbf{t}=2$. The proof of Theorem 2 follows from the theorem below in a similar way as Theorem 1 from Theorem 18 .

Theorem 19. Let $M, M^{\prime}$ be as in Theorem 2 and let $A \subset J_{0}^{4}$ be defined as in (14), and let $\Lambda_{0} \in A$. Let $j \in J$ be such that $\Lambda_{0} \in A_{j}$ and $\operatorname{dim}_{\mathbb{R}} \mathfrak{h o l}_{0}\left(\Phi_{j}\left(\Lambda_{0}\right)\right)=\operatorname{dim}_{\mathbb{R}} \mathfrak{h o l}{ }_{0}\left(M^{\prime}\right)=\ell$. Then $\Phi_{j}\left(\Lambda_{0}\right)$ is locally rigid.

Proof. The result can be proved using the properness and freeness of the action of isotropies according to the Lemmas 15, 16 and 17. This allows to employ the local slice theorem for free and proper actions and the conclusion can be obtained by arguing exactly as in the proof of Theorem 25 in [7].

\section{REFERENCES}

[1] M. S. Baouendi, P. Ebenfelt, and L. P. Rothschild, Algebraicity of holomorphic mappings between real algebraic sets in $\mathbf{C}^{n}$, Acta Math. 177 (1996), no. 2, 225-273, DOI 10.1007/BF02392622. MR1440933 (99b:32030)

[2] _ Real submanifolds in complex space and their mappings, Princeton Mathematical Series, vol. 47, Princeton University Press, Princeton, NJ, 1999. MR1668103 (2000b:32066)

[3] _ Rational dependence of smooth and analytic CR mappings on their jets, Math. Ann. 315 (1999), no. 2, 205-249, DOI 10.1007/s002080050365. MR1721797 (2001b:32075)

[4] V. K. Beloshapka and A. G. Vitushkin, Estimates of the radius of convergence of power series that give mappings of analytic hypersurfaces, Izv. Akad. Nauk SSSR Ser. Mat. 45 (1981), no. 5, 962-984, 1198 (Russian). MR637612 (83f:32017)

[5] Chung-Ki Cho and Chong-Kyu Han, Finiteness of infinitesimal deformations of CR mappings of CR manifolds of nondegenerate Levi form, J. Korean Math. Soc. 39 (2002), no. 1, 91-102, DOI 10.4134/JKMS.2002.39.1.091. MR1872584 (2002j:32036)

[6] John P. D'Angelo, Proper holomorphic maps between balls of different dimensions, Michigan Math. J. 35 (1988), no. 1, 83-90, DOI 10.1307/mmj/1029003683. MR931941 (89g:32038)

[7] Giuseppe and Lamel Della Sala Bernhard and Reiter, Local and infinitesimal rigidity of hypersurface embeddings, Trans. Amer. Math. Soc. 369 (2017), no. 11, 7829?7860, DOI 10.1090/tran/6885.

[8] James J. Faran, Maps from the two-ball to the three-ball, Invent. Math. 68 (1982), no. 3, 441-475, DOI 10.1007/BF01389412. MR669425 (83k:32038)

[9] Xiaojun Huang, On a linearity problem for proper holomorphic maps between balls in complex spaces of different dimensions, J. Differential Geom. 51 (1999), no. 1, 13-33. MR1703603 (2000e:32020)

[10] Xiaojun Huang and Shanyu Ji, Mapping $\mathbf{B}^{n}$ into $\mathbf{B}^{2 n-1}$, Invent. Math. 145 (2001), no. 2, 219-250, DOI 10.1007/s002220100140. MR1872546 (2002i:32013)

[11] Shanyu Ji, A new proof for Faran's theorem on maps between $\mathbb{B}^{2}$ and $\mathbb{B}^{3}$, Recent advances in geometric analysis, Adv. Lect. Math. (ALM), vol. 11, Int. Press, Somerville, MA, 2010, pp. 101-127. MR2648940 (2011c:32023)

[12] Robert Juhlin and Bernhard Lamel, Automorphism groups of minimal real-analytic CR manifolds, J. Eur. Math. Soc. (JEMS) 15 (2013), no. 2, 509-537, DOI 10.4171/JEMS/366. MR3017044 
[13] Bernhard Lamel, Holomorphic maps of real submanifolds in complex spaces of different dimensions, Pacific J. Math. 201 (2001), no. 2, 357-387, DOI 10.2140/pjm.2001.201.357. MR1875899 (2003e:32066)

[14] Jiř́ Lebl, Normal forms, Hermitian operators, and CR maps of spheres and hyperquadrics, Michigan Math. J. 60 (2011), no. 3, 603-628, DOI 10.1307/mmj/1320763051. MR2861091

[15] Michael Reiter, Classification of holomorphic mappings of hyperquadrics from $\mathbb{C}^{2}$ to $\mathbb{C}^{3}$, J. Geom. Anal. 26 (2016), no. 2, 1370-1414, DOI 10.1007/s12220-015-9594-6.

[16] Topological aspects of holomorphic mappings of hyperquadrics from $\mathbb{C}^{2}$ to $\mathbb{C}^{3}$, Pacific J. Math. 280 (2016), no. 2, 455-474, DOI 10.2140/pjm.2016.280.455.

[17] S. M. Webster, The rigidity of $C$ - $R$ hypersurfaces in a sphere, Indiana Univ. Math. J. 28 (1979), no. 3, 405-416, DOI 10.1512/iumj.1979.28.28027. MR529673 (80d:32022)

Department of Mathematics, American University of Beirut (AUB)

E-mail address: gd16@aub.edu.1b

FAKUltät Für MATHEMATIK, UNIVERSität WIEN

E-mail address: bernhard.lamel@univie.ac.at

FAKUltät FÜr MAthematik, Universität Wien

E-mail address: m.reiter@univie.ac.at 